\title{
Integrated control platform with load balancing algorithm in hybrid optical wireless
} networks

\author{
Yan, Ying; Dittmann, Lars; Wong, S.; Kazovsky, Leonid
}

\section{Published in:}

Proceedings NTMS

Link to article, DOI:

10.1109/NTMS.2009.5384718

Publication date:

2009

Document Version

Publisher's PDF, also known as Version of record

Link back to DTU Orbit

Citation (APA):

Yan, Y., Dittmann, L., Wong, S., \& Kazovsky, L. (2009). Integrated control platform with load balancing algorithm in hybrid optical wireless networks. In Proceedings NTMS (pp. 1-5). IEEE.

https://doi.org/10.1109/NTMS.2009.5384718

\section{General rights}

Copyright and moral rights for the publications made accessible in the public portal are retained by the authors and/or other copyright owners and it is a condition of accessing publications that users recognise and abide by the legal requirements associated with these rights.

- Users may download and print one copy of any publication from the public portal for the purpose of private study or research.

- You may not further distribute the material or use it for any profit-making activity or commercial gain

- You may freely distribute the URL identifying the publication in the public portal 


\section{Integrated Control Platform with Load Balancing Algorithm in Hybrid Optical Wireless Networks}

\author{
Ying Yan and Lars Dittmann \\ Department of Photonics Engineering \\ Technical University of Denmark \\ Lyngby, Denmark \\ e-mail:\{yiya, ladit\}@fotonik.dtu.dk
}

\author{
Shing-Wa Wong and Leonid G. Kazovsky \\ Photonics and Networking Research Laboratory \\ Department of Electrical Engineering, Stanford University \\ Stanford, CA, USA \\ e-mail: shingw@stanford.edu
}

\begin{abstract}
In this paper we cover the problem of load balancing in terms of optimum cell selection and wireless channel condition in the hybrid optical wireless network. For a given set of multimedia users with minimum quality of service requirements and a set of best effort users we find the optimum resource allocation that guarantees services for the users and maximizes the total network utility. Our model applies for the convergence network of EPON and WiMAX where the TDM-like resource assignment scheme and the WiMAX air interfaces are used. We achieve convexity of the problem and solve the optimization problem. The proposed algorithm is simulated using MATLAB and the results show validation of the framework, i.e., load balancing scheme gains better network performances.
\end{abstract}

Keywords- EPON, WiMAX, load balancing, cell breading, wireless channel condition, hybrid optical wireless networks

\section{INTRODUCTION}

The main appeal of Hybrid Optical Wireless (HOW) network is that it provides both high bandwidth and user mobility. An integration of EPON and WiMAX network is an example of the HOW network, which is a convergence of an EPON deployed as a backhaul and multiple WiMAX networks connected as the front-end networks. However, since the optical- and wireless- technologies are originally designed for difference access areas, it poses significant challenges to multiple network cooperation such as routing, resource management, and quality-of-service (QoS) provisioning, especially when multimedia services are applied. Although there are existing control schemes in both EPON and WiMAX networks, simply connecting two networks may cause limitations of network performances because of differences in their physical infrastructures.

In the hybrid network architecture, the resource management plays a key role to ensure an efficient usage of both optical link and radio spectrum. This is of particular importance for next generation broadband access networks which support data, voice and multimedia services (tri-play services) to multiple users simultaneously. To improve network performance, many hybrid network architectures and management schemes have been proposed [1]-[5] to allocate network resources efficiently and integrally, for the purpose of provisioning end-to-end QoS among both optical and wireless network layers. A system level scheme, an integrated control infrastructure, is required to be considered since most of research efforts in this field have been focused on either EPON or WiMAX solely. Our previous studies [4] shows that the hybrid network demands an integrated control plane to achieve overall and cooperated administration. Several system functions are addressed, including routing, scheduling and call admission control.

In this paper, we follow up the investigation of the integrated control platform in the hybrid EPON and WiMAX network. In particular, we explore a load balancing scheme using the cell breathing technique. The concept of cell breathing is used in conventional cellular networks to dynamically change the coverage area of cell towers [7][11]. Fully loaded cells contract their coverage area whereas the lightly loaded cells expand their coverage area. Basic idea of load balancing is to relocate users in overlapping region from fully loaded cells to lightly loaded cells. Our goal is to propose a novel integrated load balancing scheme, which utilizes a cooperative signaling protocol to collect WiMAX network status and makes centralized power assignment decisions in the EPON.

The hybrid EPON and WiMAX network architecture is illustrated in Figure 1. which delivers services to both

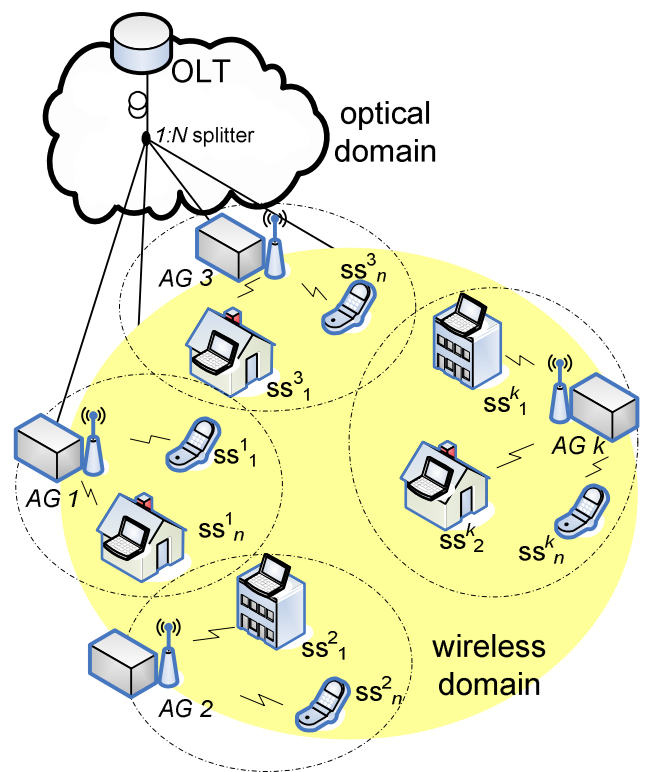

Figure 1. Hybrid EPON and WiMAX network architecture 
wireline and wireless users. In EPON, a centralized Optical Line Terminal (OLT) communicates with multiple connected Optical Network Users (ONUs) via a passive optical splitter. In the Point-to-Multipoint (PMP) WiMAX network, a Base Station (BS) manages channel allocation to Subscriber Stations (SSs) as a central controller. In hybrid network architecture, the ONU functions and BS functions are integrated into a single device, namely an Access Gateway $(A G)$, which handles connections within the wireless network, and connections cross both EPON and WiMAX network (shown in Figure 2. ).

Traditionally network control mechanisms are designed separately and exclusively in either optical networks or wireless networks. The characteristics and challenges of the hybrid network architecture are ignored. The principles of hybrid EPON WiMAX network allow the OLT to distribute data to AGs, which further relay data to the target SSs. To take advantage of the centralized system and the integrated control scheme, we propose a load balancing algorithm together with the optical downlink scheduler, which is able to control the AG transmit power dynamically and relocate traffic load in the overlapping area away from the heavily loaded cell. We introduce a new approach for the hybrid EPON and WiMAX network, where integrated control platform are deployed to manage network resources to maximize the overall system performances. A centralized power allocation control is implemented in the OLT unit. Each front-end WiMAX base station is assigned with a proper power level, in advance with a predefined power adjusting scheme, then the Signal to Interference plus Noise Ratio (SINR) dependent resource assignment is applied to achieve quality transmission. This is done by exploiting a set of suitable power profiles that derive the maximum network throughput and user quality of service.

The remainder of this paper is organized as follows. Section II introduces the system model and formulates the power allocation problem. Section III presents our proposed cell breathing scheme for load balancing. Section IV shows our simulation results and analysis. Finally, conclusion is given in Section IV.

\section{SySTEM MODEL AND PROBLEM FOMULATION}

We consider the hybrid architecture with multi-cell WiMAX frond-end network, where $K$ AGs that are connects to a central control station, OLT, via the optical link as shown in Figure 1. The wireless network consists of a set of $k=\{1, \ldots, K\}$ of cells and a set of $n=\{1, \ldots, N\}$ users in each cell. The service area is partitioned so that each Subscriber Station (SS) is connecting to only one AG at any given time. For simplicity of presentation, a system

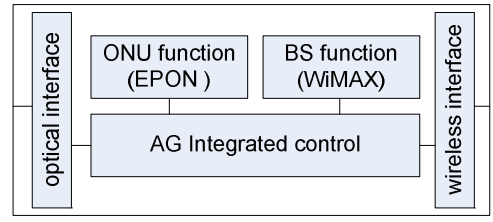

Figure 2. Integrated ONU and BS functions in AG unit consisting of two cells is used to illustrate the cell breathing technique as shown in Figure 3. The operation area is modeled by a circle with a coverage radius $\left(R_{r}{ }^{k}\right)$. At any given time $t$, the cell sizes, the number of connected SSs, and the offered data rates to $\mathrm{SS}$ are varied by allocating a different transmitting power $\left(P_{t}^{k}\right)$ to the $\mathrm{AG}^{k}$. For instance, $A G^{l}$ initially is assigned with power $P_{t}{ }^{l}$ and covers $\mathrm{SS}_{1-4}^{1}$. After $A G^{l}$ is reported as overloaded, $R_{r}{ }^{l}$ is contracted and its neighboring cell $\left(R_{r}{ }^{2}\right)$ is expanded in order to accommodate additional $\mathrm{SS}_{3-4}^{1}$. Thus, the traffic load is balanced between $A G^{l}$ and $A G^{2}$ with minimized packet loss.

Within a WiMAX cell all users share the common channel by using a TDMA radio interface. Mobility is not considered in our simulation scenario, only stationary (e.g. fixed terminals) or quasi stationary users (e.g. pedestrian) are assumed. The delivery of the packets to subscriber users is performed on a TDM frame consisting of $M$ slots. The size of cell coverage depends on transmit power, noise and path loss. The received signal at $n$th user in the $k$ th $\mathrm{AG}, \mathrm{SS}_{n}^{k}$, is the attenuated version of the transmitted power level. The propagation model includes path loss, shadowing and fading. In this paper, an Erceg propagation model defined in the IEEE 802.16 standard is used [6].

$$
\mathrm{P}_{\mathrm{n}}(d B)=20 \log _{10}\left(4 \pi \mathrm{d}_{0} / \lambda\right)+10 \gamma \log _{10}\left(\mathrm{~d}_{0} / \mathrm{d}\right)+\Delta \mathrm{PL}_{\mathrm{f}}+\Delta \mathrm{PL}_{\mathrm{h}}+\mathrm{s}
$$

Where $\lambda$ is the wavelength. $\gamma$ is the path-loss exponent. $d$ is the distance between the BS and receiver. $d_{0}$ is $100 \mathrm{~m} . \Delta \mathrm{PL}_{\mathrm{f}}$ is the frequency correction term and $\Delta \mathrm{PL}_{\mathrm{h}}$ is the receiver antenna height correction term. $s$ is a log-normal shadow fading component.

The mean received power $\left(P_{r}\right)$ is the difference between the sum of the transmit power $\left(P_{t}\right)$ and the antenna gains $\left(G_{a}\right)$ and the path loss $\left(P_{n}\right)$. The Signal to Interference plus Noise Ratio $(S I N R)$ is the ratio of received signal power to the interference signal power $\left(I_{n}\right)$, which is computed using (2). There is a minimum acceptable $\operatorname{SINR}\left(S I N R^{\text {thr }}\right)$ for receiving a quality satisfied signal.

$$
\operatorname{SINR}(d B)=\mathrm{P}_{\mathrm{r}}-\mathrm{I}_{\mathrm{n}}=\mathrm{P}_{\mathrm{t}}+\mathrm{G}_{\mathrm{a}}-\mathrm{P}_{\mathrm{n}}-\mathrm{I}_{\mathrm{n}}
$$

Where $I_{n}$ denotes the total interference received at each user, which consists of two parts: intra-cell interference and intercell interference. The intra-cell interference is caused from

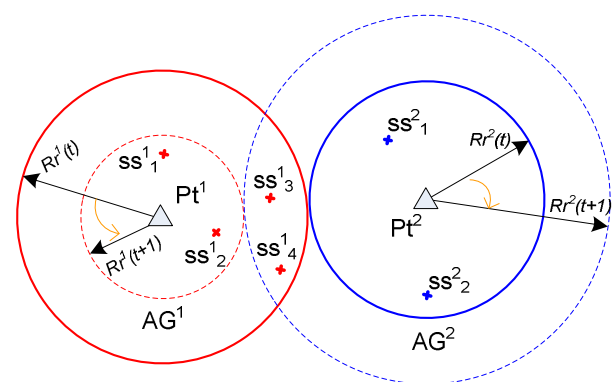

Figure 3. Cell breathing in wireless network. The solid circles represent initial coverage area (at time $t$ ) and the dotted circle represent adjusted coverage area (at time $t+1$ ). 
other SSs within the cell and the inter-cell interference is caused from the neighboring BSs. In this scenario, TDMA scheme is applied and only one SS transmits during the assigned slot time. It is assumed that there is no mutual interference among SSs in a cell. On the other hand, the initial cell coverage of $A G^{l}$ and $A G^{2}$ are non-overlapping by assigning proper transmission power values in order to avoid cross-cell interference. The theoretical system throughput of a Single Input Single Output (SISO) system, $\mathrm{AG}^{k}$, can be derived from the well-known Shannon capacity using the expression below (3):

$$
T_{\text {total }}^{k}=\sum_{\mathrm{i}=1}^{\mathrm{N}} \log _{2}\left(1+\mathrm{SINR}^{\mathrm{i}}\right), k \in K
$$

Clearly, the throughput is determined by the interference level, the channel condition, applied transmitting power and the distance between AG and SS. For simplicity, we assume that the dynamic power control at AG to allow unique data rate for individual $\mathrm{SS}$ is not utilized here. Thus, once the transmission power for the $k$ th AG during a slot time $t, P_{t}^{k}(t)$, is assigned, the system capacity can be estimated.

So we formulate the cell breathing problem as one optimization of the coverage of $A G$ and the number of served SSs. Each $\mathrm{AG}^{k}$ attains its individual fixed transmitting power lever during a time slot, $P_{t}^{k}$, to provide services with satisfied QoS constraints of each user, while optimizing the global multi-cell system throughput through balancing traffic load between AGs. Define $\mathrm{p}=\left[p_{1}, \ldots, p_{\max }\right]$ as the vector of cell power levels assigned to AGs by the OLT. Then the system problem is

$$
\operatorname{maximize} \quad \sum_{\mathrm{i}=1}^{\mathrm{K}} T_{i}\left(p_{i}\right)
$$

Subject to $\quad p_{1} \leq p_{i} \leq p_{\max } \quad \forall i \in K$,

$$
\operatorname{SINR}_{i j} \geq \operatorname{SINR}_{i j}^{t h r} \forall i \in K, \forall j \in N
$$

In the above program, the first constraint indicates that each $A G$ is assigned within a maximum power $p_{\max }$. The second constraint shows that quality transmission is ensured with assigned power. Our objective is to maximize the total throughput of the resulting power allocation.

\section{CELl BREATHING SCHEME FOR LOAD BALANCING}

EPON is a time division multiplexed (TDM) network and it relies on multi-point control protocol (MPCP) that bases on GATE and REPORT messages to grant and request for uplink bandwidth. The GATE message is broadcasted to all ONUs and target ONU receives the packets based on the labeled link layer identification (LLID). The REPORT message is reported to the OLT by ONU within its uplink transmission window. OLT allocates uplink bandwidth bases on either fixed bandwidth allocation or dynamic bandwidth allocation (DBA) algorithm. Here in the hybrid architecture, ONU functions are implemented into the AG unit.

In order to optimize the system and ensure overall QoS, we use the queue size and delay merit to determine whether or not a cell is overloaded. A cell overloading is said to occur, if the total queued data for all traffic classes at that instant exceeds a threshold, $Q_{t h r}$, or delay to transmit the queued real-time traffic is violated to the requirement, $D_{t h r}$. In the former case, the cell cannot take more traffic due to the buffer size limitation. In the latter case, the cell cannot guarantee real-time traffic with satisfied QoS requirements.

As shown in Figure 4. the cell breathing mechanism extends the MPCP control and incorporates two frames to achieve power control and load feedback. The two frames are GATE-p and REPORT-p. Similar to GATE, GATE- $p$ is broadcast downlink and contains a quantity pair (LLID and $\left.\mathrm{P}_{\mathrm{t}}\right)$ to indicate allocated power level to individual AGs. $R E P O R T-p$ is a modification of the existing REPORT message and adds the residual queue length $\left(Q_{\text {res }}\right)$ and

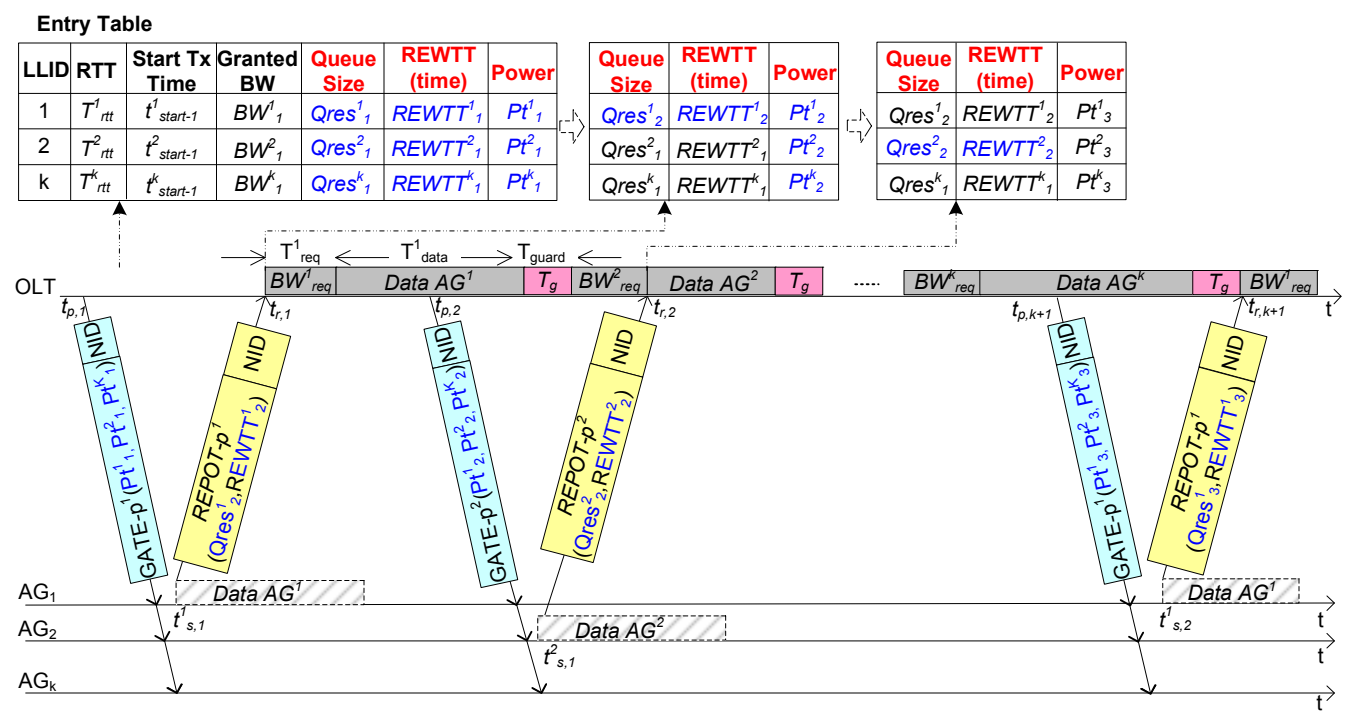

Figure 4. Extending MPCP with cell breathing algorithm 
TABLE I. FROND-END WIRELESS SYSTEM PARAMETERS

\begin{tabular}{|l|l|}
\hline \multicolumn{1}{|c|}{ Parameters } & \multicolumn{2}{c|}{ Value } \\
\hline SS distribution & Random uniform \\
\hline Frequency band & $3.5 \mathrm{GHz}$ \\
\hline System bandwidth & $10 \mathrm{MHz}$ \\
\hline Propagation model & $\begin{array}{l}\text { Erceg model; Propagation environment type } \\
\mathrm{C}: \text { flat terrain with light tree densities. }\end{array}$ \\
\hline BS power & $+40 \mathrm{dBm}$ \\
\hline fading st. dev. & $8 \mathrm{~dB}$ \\
\hline BS antenna gain & $+17 \mathrm{dBi}$ \\
\hline SS antenna gain & $0 \mathrm{dBi}$ \\
\hline BS antenna height & $35 \mathrm{~m}$ above ground \\
\hline SS antenna height & $2 \mathrm{~m}$ above ground \\
\hline Noise power & $-138.41 \mathrm{dBm}$ \\
\hline Noise figure & $7 \mathrm{~dB}$ \\
\hline
\end{tabular}

Residual Expected Wireless Transmission Time (REWTT) to the message. When DBA is employed versus fixed bandwidth allocation, REPORT-p message and the embedded downlink load information is feedback to the OLT aperiodically. In addition to avoid extended downlink traffic backlog, cell breathing can also be adjusted to relieve backlog due to poor channel conditions suffered at the AGs. To account for random wireless channel fluctuations, the feedback mechanism is important and necessary.

After receiving bandwidth requests from registered AGs, the granted bandwidth and transmission start time are assigned by the OLT without conflict. AGs are polled in sequence based on the scheduling police used in the OLT. In this example, AGs are polled in an order of their LLID number. The following cell breathing procedure is carried out in the OLT and AGs:

- At time $t_{p, 1}$, the OLT broadcasts power assignments to its connected AGs via GATE-p message. Each AG adjusts its transmitting power and associates SSs.

- $\quad$ After receiving the GATE-p message, $\mathrm{AG}^{1}$ is polled and starts its uplink transmission at $t_{s, 1}^{1}$. Along with data, the $Q_{\text {res }}$ and $R E W T T$ information are reported to the OLT. At $t_{r, 1}$, the initial entry table is updated.

- If the AG1 is sufficiently crowded, the OLT reduces the assigned power to $\mathrm{AG}^{1}\left(\mathrm{Pt}^{1}{ }_{2}<\mathrm{Pt}^{1}{ }_{1}\right)$ and increases its neighboring cell powers $\left(\mathrm{Pt}^{\mathrm{k}}{ }_{2}>\mathrm{Pt}^{\mathrm{k}}{ }_{1}\right)$. The GATE-p message is broadcasted and all AGs adjust their transmission power levels.

- $\quad$ Since the subscriber device chooses the base station with the strongest Received Signal Strength Indication (RSSI) among all received signals from AGs. When the cell coverage changes, some subscribers in the overlapping region (e.g. $\mathrm{SS}_{3}^{1}, \mathrm{SS}_{4}{ }_{4}$ in Figure 3. will be forced to handover to a less loaded neighboring cell.

- Although all AGs received the GATE-p message, only AG2 is polled at $t_{s, 1}^{2}$.

- The transmission power cannot decrease indefinitely. There is a minimum value and the power level is decreased step-wisely.

- The feedback from AG1 after decreasing its transmit power and contracting its region will be reported to the OLT at its next polling time, $t_{s, 2}^{l}$.

\section{Simulation Results}

We simulate the cell breathing scheme in MATLAB software. The EPON system is modeled as a tree topology, where an OLT connects to $K$ cells $(K=16$ or 32$)$ via optical link. The downlink optical transmission rate is $1.25 \mathrm{Gbps}$ and data are broadcasted from the OLT to AGs. In the WiMAX system, network parameters are tabulated in TABLE I. For simplicity there is one AG per wireless cell. SSs are randomly allocated within the cell region.

For the simulation study, we consider one heavily loaded cell at $\mathrm{AG}^{1}$. Few traffic arriving are designating to the neighboring cells of $\mathrm{AG}^{1}$ and thus its neighbor cells are available to load balance bandwidth from the overlapped $A G^{1}$. The traffic is generated using Poisson distribution and the SSs are equally and uniformly distributed within each cell. The simulation compares the performances of the load balancing (LB) solution against without using load balancing (NLB). In the simulation, AG drops incoming packet when either the queue is full or if the delay requirement for realtime traffic is not met.

We first simulate the network throughput rate vs. input traffic amount (shown in Figure 5. ). Input traffic amount is used instead of the input optical rate because optical line rate is significantly higher than wireless data rate. Thus, total input traffic amount is presented and the simulation generates the indicated amount of input traffic periodically over many periods. The LB solution increases the network throughput in excess of $40 \%$ at best and the improvements become more obvious when the input traffic load increases. It demonstrates that the proposed cell breathing operation can effectively handle heavy load in $\mathrm{AG}^{1}$ by reduces the transmit power of $\mathrm{AG}^{1}$ and reallocates boundary SS. In the case of NLB, $A G^{1}$ continues to serve the boundary SS, which overloads the AG while demanding relatively longer transmission time due to low SINR available at the crowded cell boundary. Hence it is advantageous to balance traffic load among cells.

Next shown in Figure 6., the dropping probability is evaluated in different scenarios with different input traffic load. Under light traffic load, the dropping probability of LB solution is zero, which performs better than the NLB solution. However, when the input traffic load continues to increase, neither LB nor NLB can meet the delay requirement, i.e. $75 \mathrm{~ms}$, for the real-time traffic. The gap between the two curves consists of the output throughput rate difference and the traffic load from the boundary area. We note that LB algorithm outperforms NLB and the amount of improvement depends on the availability of boundary SSs that can be reallocated to neighbor cells.

We also compare the scenario that 32 AGs are connected in EPON system with previous 16 AGs. When there are more AGs added, the interval between polling the same AG is increased. In other words, the period of receiving the feedback of power adjustment is increased, which may cause problem if the period is too long. However, as seen in Figure 7. LB scheme is well performed in the 32 AGs case, because 
the time slot assigned to each AG is reasonable. Thus cell

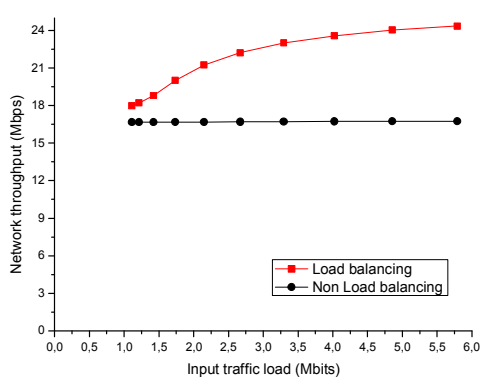

Figure 5. Network throughput enhances with cell breathing.

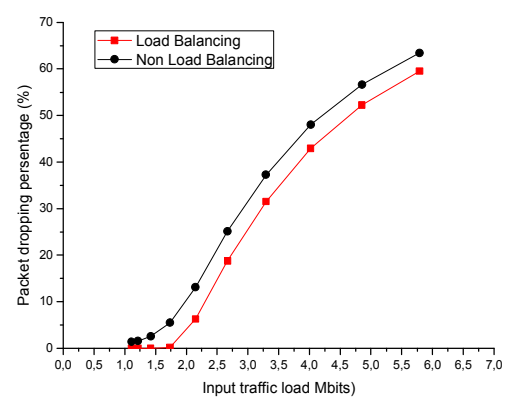

Figure 6. Packet drop probability reduces with 1 cell breathing.

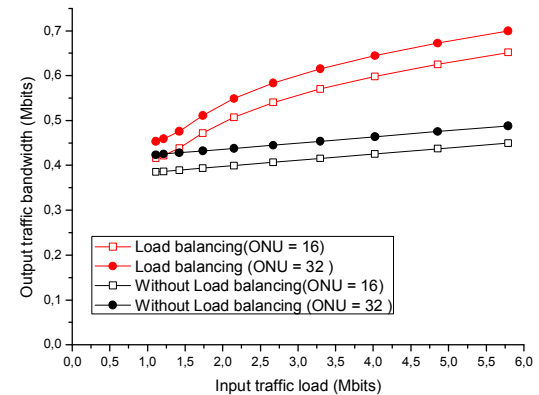

Figure 7. AG wireless output bandwidth comparison.

breathing scheme can be used with a larger number of AGs.

\section{CONCLUSIONS}

In this paper, a load balancing algorithm using dynamic power control to effectively adjust the size of cell coverage to maximize overall network capacity is proposed. In the OLT, the objective is to jointly make downlink scheduling decision and determine the transmit power.

The basic idea of this algorithm is when a cell sends out a heavily loaded indicator, the OLT starts redirecting some SSs that located in overlapping region to less loaded neighboring cells by reducing the transmit power. The term 'cell breathing' is referred to the phenomenon where the size of a cell shrinks when the load increases, and expands when the load decreases. In this way, the heavily loaded cell can shift its load in the overlap area to its neighboring cells.

This algorithm, which we refer to as the integrated load balancing problem, improves two main network performances while ensuing that users achieve QoS targets. First, it eliminates the case that a cell becomes overloaded, which reduces the probability of packet dropping and overdue delay. Second, it increases the bandwidth utilization in multi-cell system.

\section{ACKNOWLEDGMENT}

This work was supported in part by the European Committee through project ALPHA (Architectures for fLexible Photonic Home and Access networks).

This work presented is supported in part by National Science Foundation under grant no. 0627085.

\section{REFERENCES}

[1] W.-T. Shaw, S.-W. Wong, N. Cheng, K. Balasubramanian, X. Zhu, M. Maier, and L.G. Kazovsky, "Hybrid Architecture and Integrated Routing in a Highly Scalable Optical-Wireless Network," IEEE/OSA Journal of Lightwave Technology, issue 11, vol. 25, pp.3343-3351, Nov. 2007.

[2] S. Sarkar, S. Dixit and B. Mukherjee, "Hybrid wireless-optical broadband-access network (WOBAN): a review of relevant challenges", Journal of Lightwave Technology (invited paper), Vol. 25, No.11, Nov. 2007.

[3] G. Shen, R. S. Tucker, and C. Chae, "Fixed Mobile Convergence Architectures for Broadband Access: Integration of EPON and WiMAX”, IEEE Commun. Mag., vol. 45, pp. 44-50, August 2007.

[4] Y. Yan, H. Yu, H, Wessing and L. Dittmann, "Enhanced Signaling Scheme with Admission Control in the Hybrid Optical Wireless (HOW) Networks," High-Speed Networks Workshop, co-located with IEEE Infocom 2009 , Apr. 2009.

[5] K. Yang, O Shumao, K. Guild, and H.-H. Chen, "Covergence of Ethernet PON and IEEE 802.16 Broadband Access Networks and Its QoS-aware Dynamic Wavelength Allocation Scheme," IEEE Journal on Selected Areas in Communications, vol. 27, pp.101-116, Feb. 2009.

[6] IEEE Standard for Local and metropolitan area networks, Part 16: Air Interface for Fixed and Mobile Broadband Wireless Access Systems Amendment 2: Physical and Medium Access Control Layers for Combined Fixed and Mobile Operation in Licensed Bands and Corrigendum 1. IEEE Computer Society and the IEEE Microwave Theory and Techniques Society, February 2006.

[7] N. Bambos and S. Kandukuri, "Power controlled multiple access (PCMA) in wireless communication networks", IEEE INFOCOM Conference, 2000.

[8] S. Kiani and d. Gesbert, "Optimal and Distributed Scheduling for Multicell Capacity Maximization”, IEEE Transactions on Wireless Communications, Vol.7, No.1, pp.288-297, Jan. 2008

[9] P. Bahl, M.T. Hajiaghayi, K. Jain, S. V. Mirrokni, L. Qiu, and A. Saberi, "Cell breathing in Wireless LANs: algorithms and evaluations", IEEE Transactions on Mobile Computing, Vol.6, No.2, pp.164-178, Feb. 2007

[10] J.X. Qiu and J. W. Mark, "A Dynamic Load Sharing Algorithm Through Power Control in Cellular CDMA", Proceedings of Ninth International Symposium on Personal, Indoor, and Mobile Radio Communications (PIMRC'98), Vol.3, pp. 1280-1284,1998.

[11] H. Kim and A. Wolisz. "A radio over fiber based wireless access network architecture for rural areas", in Proc. 14 $4^{\text {th }}$ IST Mobile and Wireless Communications Summit, Jun. 2005. 